\title{
Política de saúde do homem: perspectivas de enfermeiras para sua implementação
}

\author{
Men's Health Policy: perspectives of nurses for its implementation \\ Política de Salud del Hombre: perspectivas de enfermeras para su implementación
}

\section{Willyane Andrade Alvarenga', Simone Santos e Silva", Maria Enoia Dantas da Costa Silva'"', Liana Dantas da Costa e Silva Barbosalv, Silvana Santiago da Rochalv}

\author{
' Universidade Federal de São Carlos, Programa de Pós-Graduação em Enfermagem (Mestranda). São Carlos-SP, Brasil. \\ "Instituto Materno Infantil de Pernambuco, Residência em Saúde da Mulher (Residente). Recife-PE, Brasil. \\ "I' Faculdade de Saúde, Ciências Humanas e Tecnológicas do Piauí. Faculdade Santo Agostinho, \\ Curso de Graduação em Enfermagem. Teresina-Pl, Brasil. \\ IV Faculdade Santo Agostinho, Curso de Graduação em Enfermagem. Teresina-Pl, Brasil.
}

Submissão: 14-09-2011 Aprovação: 11-02-2013

\section{RESUMO}

Pesquisa qualitativa, que objetivou descrever e analisar a percepção das enfermeiras da estratégia Saúde da Família acerca da importância e perspectivas de implementação da Política de Saúde do Homem. Foi realizada em julho de 2011, em Centros de Saúde de Teresina-PI, com dezesseis enfermeiras. Os dados foram coletados por entrevistas semiestruturadas; as falas foram categorizadas segundo Bardin e analisadas à luz do Interacionismo Simbólico. As enfermeiras consideram esta política importante e suas perspectivas de implementação são de apoio à iniciativa e de enfrentamento das dificuldades para a inclusão desse grupo e das relacionadas ao processo de trabalho. Traz contribuições sobre a atual situação de saúde do homem, para a assistência e consolidação dos cuidados de enfermagem.

Descritores: Cuidado de Enfermagem; Programa Saúde da Família; Saúde do Homem.

\section{ABSTRACT}

This is a qualitative research, aiming to describe and analyze the perception of nurses from the Family Health strategy on the importance and perspectives of implementation of the Men's Health Policy. It was developed in July 2011, in the Health Centers of Teresina-PI, with sixteen nurses. The data were obtained through semi-structured interviews; the speeches were categorized according to Bardin and analyzed in the light of the Symbolic Interactionism. The nurses consider this policy important and their prospects of implementation are supportive to the initiative of coping with difficulties for the inclusion of this group and related to the work process. It brings contributions on the current health situation of men and the assistance and consolidation of nursing care. Key words: Nursing Care; Family Health Program; Men's Health.

\section{RESUMEN}

Pesquisa cualitativa, con objetivos de describir y analizar la percepción de las enfermeras de la estrategia Salud de la Familia sobre la importancia y perspectivas de implementación de la Política de Salud del Hombre. Realizada en julio de 2011, en los centros de salud de Teresina-PI, con dieciséis enfermeras. Los datos fueron colectados por entrevistas semi-estructuradas; los discursos categorizados según Bardin y analizados a la luz del Interaccionismo Simbólico. Las enfermeras consideran esta política importante y sus perspectivas de implementación son de apoyo a la iniciativa y del enfrentamiento de las dificultades para la inclusión de ese grupo y las relacionadas al proceso de trabajo. Presenta contribuciones sobre la actual situación de salud del hombre, y la asistencia y consolidación de los cuidados de enfermería.

Palabras clave: Cuidados de Enfermería; Programa Salud de la Familia; Salud del Hombre.

Extraído de Trabalho de Conclusão do Curso "Percepção da enfermeira da estratégia Saúde da Família sobre a assistência à saúde do homem na fase produtiva", apresentado, em 2011, à Faculdade Santo Agostinho. Teresina-PI, Brasil. 


\section{INTRODUÇÃO}

Indicadores, no Brasil, mostram que há diferenças na esperança de vida ao nascer entre homens e mulheres e que em décadas anteriores a 1980 essas diferenças eram de aproximadamente cinco anos, elevando-se nas décadas seguintes, porém com valores não muito significativos. "Em 1980, enquanto as mulheres possuíam uma esperança de vida ao nascer de 66 anos, os homens detinham uma vida média de 59,6 anos, representando uma diferença de 6,4 anos" ${ }^{\prime \prime 1}$.

Depois de percorrido 32 anos, as mulheres passaram viver 6,7 anos a mais que os homens $(77,4$ anos, para o sexo feminino e 70,7 anos, para o sexo masculino), valores semelhantes aos da Colômbia. Em países desenvolvidos, como nos Estados Unidos e Canadá, a diferença é de aproximadamente 5 anos e em Cuba, país emergente em desenvolvimento, as mulheres vivem 3,9 anos a mais que os homens $(81,3$ para o sexo feminino e 77,4 para o sexo masculino) ${ }^{(2)}$.

Quanto aos indicadores de mortalidade masculina no Brasil, segundo a Organização Panamericana de Saúde (OPS), para cada 1000 homens 8,7 morrem, enquanto que o índice para mulheres é de 5,6. Traduzindo-se em um menor número de idosos nesse grupo. Entre as principais causas de óbitos no sexo masculino estão as neoplasias malignas (cânceres de estômago, pulmão e próstata); as doenças isquêmicas do coração; as doenças cerebrovasculares e as causas externas (suicídio, acidentes de transporte e homicídios) ${ }^{(2)}$.

Os homens expõem-se mais aos fatores de risco e num comparativo sobre as exposições a determinados fatores como o sedentarismo, tabagismo, obesidade e alcoolismo, eles apresentaram valores superiores ao das mulheres com relação ao tabagismo (44,6\% em homens e $31,9 \%$ em mulheres), a hipertensão (31\% em homens e $14,4 \%$ em mulheres) e ao alcoolismo (12,6\% em homens e 3,3\% em mulheres) $)^{(3)}$.

Envolver os homens na prática diária do autocuidado tem sido um desafio da estratégia Saúde da Família (ESF), principalmente quando se refere à adoção de um estilo de vida saudável, mas a ideia é compreender o problema no contexto de uma complexa teia de relações, envolvendo: o homem, a Unidade Básica de Saúde (UBS) e o estabelecimento do vínculo entre eles ${ }^{(4)}$.

Neste enfoque e na tentativa de acolher o homem, o Ministério da Saúde (MS), em 2008, formulou a Política Nacional de Atenção Integral à Saúde do Homem (PNAISH) que objetiva ampliar e facilitar o acesso do homem nos serviços de atenção primária, em outras palavras, visa à prevenção de doenças, suas complicações e a promoção da saúde dessa população(5).

No contexto das políticas de saúde, o enfermeiro como integrante da ESF, tem papel importante na prevenção de doenças/agravos e na promoção de qualidade de vida da população de modo geral e em especial, para os grupos mais vulneráveis, como os homens na fase produtiva.
Diante dos valores e dos diferenciais de indicadores de morbimortalidade entre os sexos, percebe-se uma situação de saúde desfavorável para os homens que precisa ser considerada e enfrentada pelos serviços de saúde. Em face dessa problemática, surgiu o interesse por este estudo que tem como objetivo descrever e analisar a percepção das enfermeiras ${ }^{1}$ da estratégia Saúde da Família acerca da importância da Política de Assistência à saúde do homem, bem como suas perspectivas para a implementação desta política.

\section{METODOLOGIA}

Este trabalho é um recorte de uma pesquisa mais ampla, que estudou a percepção da enfermeira da Estratégia Saúde da Família sobre a assistência à saúde do homem na fase produtiva. Neste, buscou-se descrever somente a percepção das enfermeiras relacionada à importância da Política Nacional de Atenção Integral à Saúde do Homem e às suas perspectivas para a implementação desta política na Estratégia Saúde da Família.

É um estudo qualitativo, exploratório, descritivo e de campo, realizado no mês de julho de 2011, em UBS situadas na Zona Norte de Teresina-PI. Os dados foram coletados por entrevistas semiestruturadas, orientadas por um roteiro de perguntas estruturadas para a caracterização dos sujeitos e abertas para buscar a percepção das enfermeiras.

Os sujeitos foram 16 enfermeiras que compõem as equipes das referidas UBS, as quais foram selecionadas pelos seguintes critérios: aceitarem participar da pesquisa assinando o Termo de Consentimento Livre e Esclarecido e estejam trabalhando na equipe, há pelo menos 1 ano, nestes Centros de Saúde. A amostra foi definida pelo critério de saturação das falas.

Dentre os entrevistados, $93,75 \%$ são do sexo feminino e, apenas, $6,25 \%$ do sexo masculino. Com relação à idade, $43,75 \%$ estão na faixa etária entre trinta a quarenta anos; $37,50 \%$ entre quarenta e um e cinquenta anos e, somente, $18,75 \%$ estão entre cinquenta e um e sessenta anos. Quanto ao tempo de formação, a maioria dos entrevistados possui mais de dez anos de graduação; $37,50 \%$ dos sujeitos possuem pós-graduação em Saúde Pública e 31,25\% em Saúde da Família e, apenas, 6,25\% referiu não possuir nenhuma pós-graduação. Quanto ao tempo de serviço na ESF, $62,50 \%$ das enfermeiras já têm de seis a dez anos de serviços prestados. Em relação ao turno de trabalho, $56,25 \%$ das enfermeiras trabalham no turno da manhã e $43,75 \%$ no turno da tarde.

Após a realização das entrevistas e de suas transcrições, na íntegra, os dados de interesse para a pesquisa foram agrupados e contextualizados em categorias temáticas semânticas, por meio da análise de conteúdo de Bardin e discutidas a partir do referencial teórico do Interacionismo Simbólico.

O Interacionismo Simbólico explora os fundamentos e causas de ações humanas e concebe que as pessoas definem

1 Neste estudo será utilizada a denominação "enfermeira(s)" para representar o sexo feminino e masculino, devido aos sujeitos participantes dessa pesquisa serem predominantemente mulheres. Porém, nas fontes bibliográficas e transcrições, quando o termo estiver no masculino, assim permanecerá. 
e agem em função de significações estabelecidas e processadas na interação social(6).

A análise de conteúdo de Bardin consiste em um conjunto de técnicas de análise das comunicações visando obter, por procedimentos sistemáticos e objetivos de descrição do conteúdo das mensagens, indicadores (quantitativos ou não) que permitam a inferência de conhecimentos relativos às condições de produção/recepção (variáveis/inferidas) destas mensagens $^{(7)}$.

Seguindo os preceitos da Ética em pesquisa, constantes da Resolução 196/96 do Conselho Nacional de Saúde que trata de pesquisa com seres humanos, o projeto deste estudo foi aprovado pelo Parecer $n^{\circ}$ 0137.0.043.000-11 do Comitê de Ética e Pesquisa da Faculdade de Saúde, Ciências Humanas e Tecnológicas do Piauí (NOVAFAPI), Teresina-PI. Para garantir o caráter sigiloso das informações, todos os depoentes foram nomeados numericamente.

\section{RESULTADOS E DISCUSSÃO}

As duas categorias empíricas surgidas das falas das enfermeiras apresentadas neste recorte tratam, a primeira, da importância por elas conferida à Política de Assistência à Saúde do Homem, destacando a sua contribuição para a redução dos índices de morbimortalidade nessa população, manutenção da estrutura familiar e redução dos fatores de risco, considerando que essa faixa etária masculina constitui uma parcela significativa no contingente populacional produtivo e político do Brasil. A segunda, das perspectivas favoráveis e desfavoráveis dessas profissionais para a implementação dessa política, conforme descritas:

\section{A. Importância conferida à Política de Saúde do Homem}

Esta categoria evidencia a percepção das enfermeiras sobre a importância dessa política, que, por meio de uma assistência direcionada ao homem, ou seja, com a prática efetiva de ações de proteção, promoção da saúde e prevenção de doenças, poderá reduzir a morbimortalidade masculina por causas preventivas e evitáveis, contribuindo para o aumento da expectativa de vida dessa população. Tal evidência pode ser observada nos fragmentos seguintes:

... com essa assistência de saúde (...) vai diminuir muito, principalmente, as mortes, (...) eles morrem mais do que as mulheres; acho que pelo menos vai melhorar a vida média do sexo masculino (D7).

É necessária essa iniciativa do Ministério da Saúde porque, se não tiver, vão aumentar os problemas ainda mais (...) a saúde deles está muito comprometida (D6).

As enfermeiras referem que a saúde do homem está bastante comprometida e isso é evidenciado pelos indicadores relativamente altos de morbimortalidade. No entanto, essas estatísticas se mantêm por conta da exposição do homem aos fatores de risco ao longo da vida e da renúncia às medidas de proteção e prevenção de agravos.
O conhecimento extraído da percepção destas enfermeiras sobre a morbimortalidade masculina é corroborado por estudos que tratam dos indicadores de vida média do homem. Estes indicadores são comparativamente menores que os da mulher em todas as regiões do Brasil ${ }^{(8)}$.

A frequência de maiores agravos e letalidade no sexo masculino devem-se mais a exposição a fatores de risco relacionados ao gênero do que ao biológico. Esses fatores podem ser socialmente determinados pelo estilo de vida, hábitos, costumes, sedentarismo, comportamento social, urbanização e nível socioeconômico ${ }^{(9)}$.

Outro enfoque ressaltado pelas enfermeiras diz respeito à realidade do homem já conhecida, o destaque para as doenças cardiovasculares e as causas externas, como os principais motivos de óbitos nesse grupo. Esta afirmação está presente nos fragmentos das falas seguintes:

... há muitas mortes por doenças cardiovasculares, coisas que podem ser modificáveis com o estilo de vida (...) fora os acidentes automobilísticos que nós poderíamos, também, ajudá-los, (...) sobre a forma de prevenir (D9).

... é uma boa medida tomada pelo Ministério, (...) na fase produtiva dos homens, há muitos óbitos (...) porque também é uma fase que morre muitas pessoas de acidente, tanto acidente de trabalho, como acidente de trânsito (D14).

Ressaltam que as doenças cardiovasculares, bem como as causas externas são importantes no agravamento da morbimortalidade masculina e poderiam ser evitadas com a modificação no estilo de vida dos homens. Elas acreditam que as medidas assistenciais e educativas direcionadas a esse grupo, adotadas pelo Ministério da Saúde, poderiam contribuir para a modificação dessa panorâmica. Por outro lado, enfatizam que as enfermeiras são agentes importantes na execução dessas medidas.

Destacam os acidentes de trânsito e acidentes de trabalho como causas externas de elevada incidência e possíveis de prevenção, ratificando os dados apresentados por estudos que mostram os acidentes de transporte, como segunda principal causa de morte entre os homens, perdendo apenas para a violência (principalmente homicídios) que é a primeira causa de morte. Estas são seguidas das doenças relacionadas ao apareIho digestivo (principalmente cirrose hepática) e as neoplasias malignas (predominando câncer de pulmão, próstata e estômago). Outras causas com acentuada sobremortalidade são os transtornos mentais e comportamentais, muitas vezes relacionada ao uso de álcool e substâncias psicoativas ${ }^{(8)}$.

O mesmo estudo ressalta que nas internações hospitalares, as principais causas são as doenças relacionadas ao aparelho respiratório, seguindo-se das doenças do aparelho circulatório, infecciosas e parasitárias, do aparelho digestivo e lesões. Além disso, esse grupo masculino é o principal autor de roubos e assaltos e os protagonistas na violência doméstica, o que reflete na maior população penitenciária ${ }^{(8)}$.

Nesta categoria, também se evidencia que as enfermeiras percebem que a Política de Assistência à Saúde do Homem 
é importante porque poderá contribuir para a manutenção da estrutura familiar, não apenas pela existência do homem no meio da família, dado pelo prolongamento de sua vida, mas pela possibilidade de reduzir os agravos à sua saúde possibilitando com que ele viva com qualidade. Isso pode ser percebido na fala seguinte:

... as famílias ficariam muito desestruturadas, os homens adoecendo e morrendo cedo ficariam muitas viúvas e a gente sabe que é muito importante a presença masculina em uma família, em um lar (D16).

As enfermeiras destacam que o homem exerce um papel importante na família e por essa razão ela se torna desestruturada quando esse membro não está mais presente, da mesma forma que o seu adoecimento traz sofrimento e expectativas para que o homem retorne a sua posição como o líder dos familiares. Se o homem adoece toda a família é influenciada. Esta afirmação está em consonância com o estudo que aborda que o homem tem um papel de provedor na família, característica da identidade masculina, reafirmando a importância das responsabilidades com a família e com a manutenção da casa por representar o chefe do $\operatorname{lar}^{(10)}$.

As enfermeiras fazem menção ainda a contribuição que a Política Nacional de Atenção Integral à Saúde do Homem trará para a redução dos fatores de risco. Essa política se faz importante por está diretamente relacionada com a esperança e qualidade de vida do homem, uma vez que, sendo executada, atuará na proteção de sua saúde, reduzindo, abrandando ou eliminando as exposições a fatores de risco, além de estimulá-los a adotar medidas de prevenção primária à sua saúde:

... seria melhor se a gente pudesse estar orientando, quanto à questão da alimentação, estilo de vida (...) então você pode estar mudando uma série de coisa, como a questão das dislipidemias (D3).

Segundo as enfermeiras os homens se expõem mais aos fatores de risco e por isso a implementação dessa política será ideal na redução desses fatores. Sobre este assunto, estudos mostram que o homem gera fatores de risco danosos a sua saúde, resultando em doenças, lesão e morte. Investir na prevenção das doenças é garantir qualidade de vida ao homem, evitar internações hospitalares, além de gastos aos sistemas de saúde. Essas consequências resultantes das medidas de prevenção relacionam-se com quase todas as doenças de causas conhecidas e é mais efetiva e segura que a procura por indivíduos de alto risco, embora também deva ocorrer ${ }^{(3)}$.

Há muitos agravos à saúde do homem relacionados aos fatores culturais e sociais que também poderiam ser evitados, na percepção das enfermeiras, se estes procurassem as medidas de prevenção primária, modificando o seu estilo de vida como por exemplo o abandono de vícios e hábitos (fumo, álcool, estresse, trabalho, sedentarismo, entre outros) muitas coisas mudariam. Autores concordam que esses comportamentos de risco nos homens, como eles se cuidam e percebem seu corpo, têm que ser visto sob a ótica de gênero, como homens concretos relacionando-se com as masculinidades ${ }^{(11)}$.

Quanto ao risco para as doenças sexualmente transmissíveis (DSTs), as enfermeiras expressam, que a implementação dessa política diminuirá a exposição do homem a essas doenças, contribuindo para a redução da morbidade tanto masculina como feminina, pois as ações educativas a serem desenvolvidas no contexto da política, sensibilizarão esse grupo para a diminuição dos fatores de riscos:

... com relação às DSTs, eles não querem se precaver(...) não querem usar preservativo porque dizem que dói, que arde, é ruim fazer sexo com camisinha, então eu acho que tem que ter esse programa de saúde (D6).

O homem, além de ser responsável pelo seu adoecimento, ainda adoece a mulher, tornando-se obstáculo para a saúde dela, quando não adere às medidas de prevenção como o uso de preservativos, expondo a si e a parceira às DSTs. Isso se deve a uma visão machista de que cabe à mulher a responsabilidade de promoção à saúde e prevenção das doenças.

As enfermeiras ressaltam, ainda, que os homens negam o uso do preservativo, pois afirma que dói e arde, trazendo desconforto. Autores apontam que fatores como esses, relacionados ao imaginário masculino, de que o preservativo diminui o prazer sexual ou interrompe a relação e o contato direto entre o casal contribui para o seu não uso, além de que o seu uso em relacionamentos estáveis pode ameaçar a confiança entre o casal, por suspeitar de infidelidade ao crer na possibilidade de relações extraconjugais ${ }^{(12) .}$

As enfermeiras consideram que a assistência à saúde dos homens, nessa faixa etária, seja essencial por protegê-los da morte, uma vez que representam uma parcela significativa da população produtiva do país e, portanto, merecedora de cuidados:

... é muito importante, porque o óbito de homens repercute em tudo, financeiramente no país, na renda de modo geral. Eles são a força produtiva do país (D12).

As enfermeiras reafirmam a representatividade dessa população em meio ao contingente populacional por ser geradora de renda para sua casa e para o país. Apesar de hoje a mulher estar mais inserida no mercado de trabalho, ainda é o homem que compõe o maior grupo de trabalhadores no país na realidade brasileira. A faixa etária dos 25 aos 59 anos, privilegiada pela política, compõe a fase produtiva da vida do homem e o MS completa isso, afirmando que esse grupo etário masculino corresponde a 41,3\% da população masculina com diferentes fases da vida ou a $20 \%$ do total da população do Brasil, correspondendo à supremacia da força produtiva, e além do mais exerce um significativo papel sociocultural e político ${ }^{(5)}$.

Nessa categoria, a percepção das enfermeiras aponta para a importância de uma política que contemple o homem na sua integralidade, considerando as diferenças com relação ao seu estilo de vida, sua inserção na força de trabalho, entre outros aspectos, de modo a tentar reduzir os agravos à sua saúde. Nesse sentido, corroboram com o MS quando trata da 
heterogeneidade das possibilidades de ser homem, levando em consideração idade, condições socioeconômicas, situação carcerária, deficiência física e/ou mental, orientações sexuais, identidades de gênero não hegemônicas. Tudo para ser equânime na atenção a essa população ${ }^{(5)}$.

A percepção das enfermeiras, ainda coaduna com a fala do ex-ministro da saúde José Gomes Temporão nomeado por Luiz Inácio Lula da Silva, que expressa a importância de ações voltadas ao homem, destacando sua contribuição para obtenção de melhores resultados em programas que busquem a prevenção das DSTs, o controle da violência de gênero, a saúde reprodutiva e a redução do sofrimento de homens e mulheres e a garantia da cidadania(13). "Uma ação educativa bem feita 'modernizaria' os homens brasileiros, dissipando o pensamento mágico que os (des)orienta e que os torna presas de seu próprios preconceitos", pois os tornaria conscientes de sua vulnerabilidade ${ }^{(14)}$.

\section{B. Perspectivas para a implementação da Política de Saú- de do Homem na ESF}

Esta categoria evidencia a percepção dos enfermeiros sobre as perspectivas favoráveis e desfavoráveis para a implementação da Política de Assistência ao Homem na ESF. Nos fragmentos das falas dessas enfermeiras, observou-se o que elas esperam pela implementação e expressam suas ideias para que a prática da assistência à saúde do homem aconteça, em que os profissionais e os serviços de saúde devem melhorar para que efetivamente esta assistência torne-se realidade.

Iniciativa perfeita a do Ministério da Saúde (...) chegou a hora de não só vermos o homem como o hipertenso (...), o diabético, mas com as prováveis e possíveis doenças que podem acometê-lo e que podem ser prevenidas (...) cada faixa etária requer um cuidado diferenciado e eu acho que o homem também merece (D3).

... a saúde do homem em si (...) a gente vai deixando para lá, mas a gente tem que voltar os olhos para isso. A política é bastante viável, eu acredito que dá certo porque a gente tem que trabalhar com a prevenção no PSF (...) e eu acho que vale a pena, é um programa que veio para ficar (D14).

As enfermeiras apóiam a iniciativa ministerial de assistir ao homem adulto, uma vez que ele possui sua singularidade e é acometido por diversas doenças que podem ser prevenidas. Além de merecer um cuidado direcionado, levando em consideração a relação de gêneros, assim como as outras faixa etárias da população. Segundo elas, isso é realizar uma assistência integral e equânime, entendendo o processo saúde-doença no homem.

Estudos têm sido realizados com o objetivo de entender o processo saúde-doença do homem, considerando suas diferentes características (raça/cor, etnia, classe social, entre outras). Discussões acerca da promoção da saúde, dos direitos e da equidade ganharam destaque nos dias atuais, contribuindo para a saúde do homem sob a ótica das masculinidades e permitindo que sejam vistos em suas singularidades ${ }^{(8)}$.
Conforme as enfermeiras, o homem ainda é visto, somente quando está acometido por alguma morbidade (hipertensão, diabetes, cardiopatias, câncer, outras), sendo esta situação associada à sua atitude de rebeldia perante as ações de saúde principalmente, quando estas são de prevenção e promoção. É hora dos homens se sensibilizarem em relação ao seu papel de eternos provedores e "fortaleza sem brechas", de reverem o seu comportamento e modificarem suas condutas, adentrando o Sistema Único de Saúde (SUS) pela atenção básica ${ }^{(1)}$.

As enfermeiras consideram a prática dessa assistência viável e percebem a filosofia da atenção básica pautada em ações de promoção/ proteção da saúde e prevenção e não, apenas, no modelo curativo. Esta percepção está de acordo com as diretrizes da Política Nacional de Atenção Integral à Saúde do Homem, que entende a saúde do homem como um conjunto de ações de promoção e prevenção, assistência e recuperação, executadas com humanização e qualidade, nos diferentes níveis de atenção( ${ }^{(5)}$.

Com relação aos aspectos a serem considerados pelos profissionais e pelos serviços de saúde, as enfermeiras ressaltam que eles precisam ser revistos, no sentido de tornar a assistência à saúde do homem realidade:

... essa política vai dar certo porque todos os outros programas, como hipertenso e diabético - HIPERDIA dão (...). Se instituir uma coisa ligada com a família, a escola, todo mundo voltada para aquilo (...) só nós para resolvermos isso aí não dá. Só acredito que dará certo se for todo mundo (D8).

... se fosse um atendimento alternativo, limitado, um dia a noite, poderia ser que a aderência deles, pudesse ser bem maior (...) essa temática deve ser trabalhada, não de uma forma imposta, mas uma forma a se adequar a cada realidade, então muitas vezes o serviço deve se adequar a população (D10).

As enfermeiras ressaltam que para a efetividade dessa política é necessária a integração de todos os segmentos sociais: escola, igreja e, principalmente, a família, de modo a incentivar a procura do homem pela atenção básica e para o autocuidado.

Elas propõem a necessidade de definição de novos horários e turnos de funcionamento das UBS, com atendimento noturno, por exemplo, para facilitar a adesão desse grupo. Esta mudança promoveria uma adequação das ações assistenciais às demandas dessa população. Neste sentido, a percepção dessas enfermeiras condiz com o objetivo da Política de Saúde do Homem que propõe a facilidade do acesso às ações e os serviços de assistência integral à saúde ${ }^{(5)}$.

Por outro lado, esta categoria traz pontos de vista que expressam a desestimulação para a implementação da assistência à saúde do homem na fase produtiva. As falas seguintes revelam dificuldades relacionadas a três fatores principais: sobrecarga de trabalho da enfermeira pela falta de colaboração dos outros integrantes da equipe, a sensibilização dos homens para buscarem os serviços de saúde, em especial a Atenção Básica e o despreparo técnico para a utilização dos protocolos da política: 
... de não termos treinamentos, protocolos, isso favorece a dificuldade. Às vezes eu quero atuar mais, quero ir para a área trabalhar mais a questão educativo, mas me sinto limitada porque tem tanta papelada, tanta burocracia que a gente acaba dando prioridade para aquele grupo que a gente tem mais costume de trabalhar: idosos, crianças, hipertensos, diabéticos (D9).

... então, as dificuldades são imensas, imensas e a gente não sabe se vai ter êxito nesse trabalho que a gente está tentando organizar (...) são dificuldades que a gente vai enfrentar para formar outra mentalidade nos homens (D1).

As falas das enfermeiras ratificam estudos esclarecedores das dificuldades vivenciadas por essas profissionais no decorrer do tempo. Mostram nesses estudos que o enfermeiro assumiu muitas responsabilidades relacionadas à administração, à gestão do cuidado, à participação comunitária, além daquelas próprias dos burocratas do setor saúde e atualmente a quantidade de atividades/responsabilidades que o enfermeiro exerce na ESF ultrapassa os limites naturais do ser humano e do trabalho da Enfermagem. Esse número de atividades, cada vez maior, tem diminuído a qualidade de vida do enfermeiro no trabalho, pois ele desenvolve um papel profissional de "cuidador clínico-assistencial, gerente de pessoal, do território, das ações, do serviço, dos insumos, do transporte, sistematizador de estatísticas e de produção, analista de dados e de Sistema de Informação em Saúde, além da articulação política, social e comunitária" (15).

Outros estudos reconhecem o enfermeiro como efetivador das políticas de saúde, uma vez que ele representa mais de $80 \%$ do efetivo que atua nos programas de assistência de saúde pública ${ }^{(16)}$. Portanto, para haver mudanças em relação à saúde do homem ele tem que está envolvido, pois sua participação efetiva recriará mudanças nessas práticas de saúde. Este profissional precisa sentir-se importante e valorizado no seu processo de trabalho para que ele se perceba como um dos principais agentes para estabelecer o vínculo do homem com a atenção básica.

Com relação às dificuldades de sensibilizar o homem para a busca pelo serviço de saúde, a fala das enfermeiras se respalda em estudos que afirmam ser a participação do homem nas ações de saúde, no mínimo, um desafio, devido à socialização do homem não evolver cuidados com a própria saúde e cuidados com o outro ${ }^{(17)}$.

Neste sentido, é necessário que os profissionais de saúde estejam atentos a essas barreiras que dificultam o homem a buscar os serviços, também, devendo se atentar quanto à heterogeneidade das possibilidades de ser homem, considerando que essa dificuldade está principalmente, associada à masculinidade e esta é construída historicamente e socioculturalmente ${ }^{(18)}$ e ao realizar as práticas de educação em saúde deve-se realizar continuamente para que haja mudança de comportamento, caso contrario, haverá falta de estímulo da equipe de saúde para o desenvolvimento de cidadania e participação nas atividades desenvolvidas pela $U \mathrm{BS}^{(19)}$.
Quanto ao despreparo das enfermeiras para assistirem a esse grupo, elas explicitam em suas falas, a necessidade de definição de protocolos de execução da Política de Saúde do Homem e que sejam treinadas para a sua aplicação. A política se apresenta, claramente, em documentos que trazem princípios, diretrizes, objetivos, papéis dos órgãos ou setores (responsabilidades institucionais das três esferas do governo - União, Estado e Município), metas e ações prioritárias que norteiam a execução dessa política. No entanto, são válidos os relatos das enfermeiras ao apontarem a inexistência de um protocolo, que são atribuições técnicas de cada membro da equipe, planos, projetos, programas, atividades concretas ${ }^{(3,20)}$.

Sobre esse assunto, vale ressaltar, que um dos princípios da Política Nacional de Atenção à Saúde do Homem é capacitar tecnicamente os profissionais de saúde para o atendimento ao homem e cabe a União propor, em parceria com a Secretaria de Gestão do Trabalho e Educação na Saúde, estratégias de educação permanente dos trabalhadores do SUS, voltada para essa política. Assim, o MS definiu um plano de ação para um período de 2009 a 2011, tendo como eixo de ações prioritárias a qualificação de 32 mil equipes da ESF até o final de $2011^{(20)}$, certamente, as enfermeiras ainda não tinham sido envolvidas.

\section{CONCLUSÃO}

A partir dos resultados alcançados, o estudo mostra que as enfermeiras acreditam que os homens não somente merecem como precisam ser assistidos pelos programas de saúde que buscam a redução dos índices de morbimortalidade e da exposição destes aos fatores de riscos, fortalecendo a manutenção da estrutura familiar, e também, por eles constituírem uma parcela significativa da população, pois, a faixa etária masculina contemplada pela política forma a força produtiva e política do país.

A percepção das enfermeiras do estudo é clara quanto à necessidade de assistir aos homens integralmente, considerando sua heterogeneidade, atentando-se para o seu estilo de vida, de modo a buscar a redução dos agravos que eles causam à própria saúde e promover a sua longevidade com qualidade.

Apontam o desestímulo e as dificuldades para a implementação dessa política, que devem ser consideradas para se entender como essas profissionais aceitam, recusam ou transformam as ações propostas pela área da saúde. Portanto, conhecer a percepção das enfermeiras, a quem é atribuída essa atividade de saúde e sintonia com o homem, é válido, uma vez que são as enfermeiras quem irão incorporar essa política nas suas práticas de cuidar na ESF e as ações em saúde partem dessa compreensão.

Em face do exposto, os objetivos deste estudo foram alcançados e os resultados apresentados são relevantes na medida em que podem trazer contribuições para nortear o planejamento da implementação das ações de assistência ao homem tanto na realidade do estudo quanto na realidade brasileira.

Descrever a percepção das enfermeiras relacionada à importância da política de assistência ao homem, às perspectivas favoráveis e desfavoráveis para a sua implementação, bem 
como, a sensibilização destas profissionais para assumirem a execução dessas ações é de fundamental importância. Por meio deste diagnóstico, poderão as autoridades de saúde, os profissionais da área, os familiares e o próprio homem, refletir sobre novos caminhos para a efetivação dessa política e, consequentemente, a melhoria da qualidade de vida desse homem, ao mesmo tempo, que se consolidará a prática assistencial das enfermeiras na realidade do país.

\section{REFERÊNCIAS}

1. Braz MA. Construção da subjetividade masculina e seu impacto sobre a saúde do homem: reflexão bioética sobre justiça distributiva. Ciênc Saúde Coletiva 2005;10(1):97104.

2. Organización Panamericana de la Salud/Organización Mundial de la Salud (OPS/OMS). Información y Análisis de Salud (HSD/HA): situación de Salud en las Américas: Indicadores Básicos 2012. Washington; 2012.

3. Ministério da Saúde, Secretaria de Políticas de Saúde, Departamento de Ações Programáticas Estratégicas. Plano de reorganização da atenção à hipertensão arterial e ao diabetes mellitus: hipertensão arterial e diabetes. Brasília: Ministério da Saúde; 2001.

4. Figueiredo W. Assistência à saúde dos homens: um desafio para os serviços de atenção primária. Ciênc Saúde Coletiva 2005;10(1):1059.

5. Ministério da Saúde, Secretaria de Atenção à Saúde, Departamento de Ações Programáticas Estratégicas, Política nacional de atenção integral à saúde do homem: princípios e diretrizes. Brasília; 2008.

6. Charon M. Symbolic interactionism: an introduction, an interpretation, an integration. Califórnia: Editora Englewood Cliffs; 1985.

7. Bardin L. Análise de conteúdo. Lisboa: Edições 70; 2002.

8. Laurenti R, Mello Jorge MH., Gotlieb SL. Perfil epidemiológico da morbimortalidade masculina. Ciênc Saúde Coletiva 2005; 10(1):3546.

9. Laurenti R, Buchalla CM, Mello Jorge MHP, Lebrão ML, Gotlieb SLD. Perfil epidemiológico da saúde masculina na Região das Américas. Uma contribuição para o enfoque de gênero. São Paulo: Faculdade de Saúde Pública USP; 1998.

10. Figueiredo W S, Schraiber LB. Concepções de gênero de homens usuários e profissionais de saúde de serviços de atenção primária e os possíveis impactos na saúde da população masculina. [homepage na internet]. [acesso 08 maio 2010]. Departamento de Medicina Preventiva da Faculdade de Medicina da USP; 2008. Disponível em: < http://www.abrasco.org.br/cienciaesaudecoletiva/artigos/artigo_int.php?id_artigo $=3153>$

11. Schraiber LB, Gomes R, Couto Mt. Homens e saúde na pauta da Saúde Coletiva. Ciênc Saúde Coletiva 2005;10(1):717.
12. Madureira VSF, Trentini M. Relações de poder na vida conjugal e prevenção da AIDS. Rev Bras Enferm [periódico na internet]. 2008 setout [acesso 21 ago 2010];61(5):63742 Disponível em: < http://www.scielo.br/pdf/reben/v61n5/ a17v61n5.pdf $>$

13. Fonseca J L. Homens, feminismo e direitos reprodutivos no Brasil: uma análise de gênero no campo das políticas públicas (20032006). Recife. Tese [Doutorado em Saúde Pública]Departamento de Saúde Coletiva; Centro de Pesquisas Aggeu Magalhães; Fundação Oswaldo Cruz; 2008. p. 262.

14. Carrara S, Russo JA, Faro L. A política de atenção à saúde do homem no Brasil: os paradoxos da medicalização do corpo masculino. Physis Rev Saúde Coletiva 2009;19(3):65978.

15. Ximenes FR, Neto, Ponte MA, Vasconcelos do Amaral MI, Rocha J, Cunha IC. Percepção dos enfermeiros sobre a prática profissional na estratégia saúde da família. Biblioteca Lascasas. [homepage na internet]. [acesso 16 maio 2010];5(5) Disponível em: <http://www.indexf.com/lascasas/documentos/lc0468.php $>$

16. Araújo MFS, Oliveira FMC. A Atuação do Enfermeiro na Equipe de Saúde da Família e a Satisfação Profissional. CAOS - Rev Eletrônica Ciênc Soc [periódico na internet]. 2009 set [acesso 10 maio 2010];1(14):314 Disponível em: < http://www.cchla.ufpb.br/caos>

17. Keijzer B. Hasta donde el cuerpo aguante: género, cuerpo y salud masculina. In: Cáceres CF, Cueto M, Ramos $M$, Vallas $S$, editores. La salud como derecho ciudadano: perspectivas y propuestas desde América Latina. Lima: Universidad Peruana Cayetano Heredia; 2003. p. 13752.

18. Pirolo SM, coordenador. O desafio de operacionalizar as ações de Atenção Integral à Saúde do Homem na Estratégia Saúde da Família. Marília; 2009.

19. Costa GD, Mitre Cotta RM, Ferreira MLS, Reis JR, Franceschini SDC. Saúde da família: desafios no processo de reorientação do modelo assistencial. Rev Bras Enferm [periódico na internet]. 2009 janfev [acess0 21 ago 2010];62(1):1138. Disponível em: < http://www.scielo. br/pdf/reben/v62n1/17.pdf>

20. Ministério da Saúde, Secretaria de Atenção à Saúde. Departamento de Ações Programáticas Estratégicas. Plano de ação nacional (20092011) da Política Nacional de Atenção Integral à Saúde do Homem. Brasília: Ministério da Saúde; 2009. 